\title{
A review of the presentation and outcome of takotsubo cardiomyopathy in COVID-19
}

\author{
Kevin John', Amos Lal' ${ }^{2}$, Ajay Kumar Mishra ${ }^{3}$ \\ ${ }^{1}$ Department of Critical Care, Bangalore Baptist Hospital, Hebbal, Bengaluru, Karnataka, India; ${ }^{2}$ Department of Medicine, \\ Division of Pulmonary and Critical Care Medicine, Mayo Clinic, Rochester MN, USA, ${ }^{3}$ Department of Medicine, Division \\ of Cardiology, Saint Vincent Hospital, Worcester, MA, USA
}

\begin{abstract}
Although the most frequent presentation of the novel Coronavirus disease-2019 (COVID-19) is a respiratory syndrome, cardiac involvement is being increasingly recognized. One such entity is takotsubo cardiomyopathy. We sought to review the various cases of takotsubo cardiomyopathy reported during the COVID-19 pandemic and consolidate the information available on its clinical features, evaluation and treatment. We performed a PubMed search using the MeSH terms "Takotsubo Cardiomyopathy" or "Stress Cardiomyopathy" and "COVID-19", and identified 16 case reports, two case series, and one retrospec-
\end{abstract}

Correspondence: Dr. Kevin John, Senior Resident, Department of Critical Care, Bangalore Baptist Hospital, Bellary Road, Hebbal, Bengaluru, Karnataka, India 560024.

E-mail: kevinjohn619@gmail.com

Key words: COVID-19; Takotsubo cardiomyopathy; cardiomyopathy

Contributions: KJJ and AKM contributed to the conceptual design of the study. KJJ and AKM independently screened the articles and extracted the data. KJJ, AKM and AL contributed to write-up and submission of the study. AKM and AL reviewed the final manuscript. All authors reviewed and agreed with the final content of the article.

Conflict of interest: The authors declare that they have no competing interests, and all authors confirm accuracy.

Availability of data and material: All the data that was used is included within the manuscript.

Ethics approval and consent to participate: Not applicable.

Consent for publication: Not applicable.

Received for publication: 30 November 2020 .

Accepted for publication: 23 January 2021.

COCopyright: the Author(s), 2021

Licensee PAGEPress, Italy

Monaldi Archives for Chest Disease 2021; 91:1710

doi: 10.4081/monaldi.2021.1710

This article is distributed under the terms of the Creative Commons Attribution Noncommercial License (by-nc 4.0) which permits any noncommercial use, distribution, and reproduction in any medium, provided the original author(s) and source are credited. tive cohort study. There was a total of 24 reported patients with COVID-19 infection, who developed takotsubo cardiomyopathy, and two patients without COVID-19 who developed takotsubo cardiomyopathy due to the emotional stress associated with the global pandemic. The mean age of the patients was 67.19 years (SD 15.83) and $16(59.3 \%)$ were women. Chest pain was reported in only ten patients $(38.46 \%)$ and ST-elevation was seen in 11 patients $(42.3 \%)$. While most patients had typical takotsubo cardiomyopathy, four patients had inverted (reverse) takotsubo cardiomyopathy, two had bi-ventricular involvement, one had median takotsubo and another had global takotsubo with apical sparing variant. Most patients had a positive outcome with complete or near-complete reversal of cardiac dysfunction at the time of discharge. Five deaths (19.23\%) were reported. Takotsubo cardiomyopathy is a rare, but increasingly reported reversible cardiomyopathy that can be seen in patients with COVID-19 infection and the diagnosis must be actively sought for in these patients.

\section{Introduction}

Coronavirus disease 2019 (COVID-19) emerged from China in December of 2019 and continues to be a public health emergency of international concern. As of November 10, 2020, severe acute respiratory syndrome coronavirus 2 (SARS-CoV-2) has infected 50.8 million individuals worldwide and has caused more than 1.26 million deaths. COVID-19 can be asymptomatic or symptomatic. The incubation period is generally within 14 days following exposure, and most symptomatic infections occur approximately four to five days after exposure $[1,2]$. The most common symptoms in decreasing order of occurrence are cough, fever, myalgia, headache, dyspnea, sore throat, diarrhea, nausea or vomiting, loss of smell, abdominal pain and rhinorrhea [3]. As our understanding of this novel pathogen improves and more cases are reported, unusual and rare presentations associated with COVID19 are being recognized.

Takotsubo cardiomyopathy (also known as transient apical ballooning, stress cardiomyopathy and 'broken heart syndrome) is a unique reversible form of cardiomyopathy which presents with symptoms and signs mimicking acute myocardial infarction, without actual coronary artery stenosis, thrombosis or spasm $[4,5]$. The name is derived from the shape the heart takes, which resembles a Japanese octopus fishing pot called a 'takotsubo'. Takotsubo cardiomyopathy occurs predominantly in postmenopausal women in the setting of extreme physical or emotional stress. There have been reports of increased incidence of takotsubo cardiomyopathy after natural calamities, such as what was observed during the 
Niigata earthquake when the incidence of takotsubo cardiomyopathy was 24-fold higher near the epicenter, one month after the earthquake [6]. It has been estimated that $1-2 \%$ of all patients presenting with an initial diagnosis of acute coronary syndrome have takotsubo cardiomyopathy [5].

Although a respiratory syndrome is the predominant clinical presentation of COVID-19, cardiac involvement is being increasingly recognized. One such cardiac complication is takotsubo cardiomyopathy. In this article, we review the various cases of takotsubo cardiomyopathy reported during the COVID-19 pandemic and attempt to consolidate the available information on its clinical features, evaluation and treatment.

\section{Methods}

In this review, we included articles on COVID-19 and takotsubo cardiomyopathy published in PubMed. We used the search terms "COVID-19" and "Takotsubo Cardiomyopathy" or "Stress Cardiomyopathy" under the Mesh database in PubMed. Case reports, case series, retrospective, and prospective observational studies on adult patients with COVID-19 were eligible to be included. We excluded opinions, recommendations and reviews which did not include clinical details of patients. Two independent clinicians were involved in the screening of the articles.

\section{Results}

We identified 22 articles on "Takotsubo Cardiomyopathy" or "Stress Cardiomyopathy" and "COVID-19" published in PubMed till $10^{\text {th }}$ November 2020 . One article was a review of the cardiovascular manifestations of COVID-19, and was excluded as there were no patient details in the study. Two papers which were commentaries were also excluded. Sixteen case reports, two case series, and one retrospective cohort study that investigated the incidence of takotsubo cardiomyopathy during the SARS-CoV-2 pandemic were included in the review. A case report which was in Italian was translated using Google translator.

Among the 16 case reports and 2 case series, there were a total of 24 patients with COVID-19 infection, who developed takotsubo cardiomyopathy [7-22]. Twenty-three of these patients had COVID-19 infection confirmed by reverse-transcriptase polymerase chain reaction (RT-PCR) test and one patient had serological evidence of acute infection. Also, there were two patients who had features of takotsubo cardiomyopathy, without evidence of COVID-19 infection [7,8]. Both of them had reported emotional stress as a result of the COVID-19 pandemic and social isolation, which presumably lead to the development of takotsubo cardiomyopathy. All 26 patients were included in the final analysis.

\section{Clinical presentation}

The mean age of the patients was 67.19 years (SD 15.83) and $16(59.3 \%)$ were women (Supplementary Table 1). Chest pain was reported in only ten patients $(38.46 \%)$ (Figure 1$)$. The other presenting symptoms were fever, shortness of breath, cough, fainting and diarrhea. Two patients presented with cardiac symptoms alone, and the diagnosis of COVID-19 was made after a screening test was sent $[9,10]$. Another patient was initially diagnosed with pericardial effusion in tamponade, and developed features of takotsubo cardiomyopathy after admission to the hospital [11]. Two others were diagnosed with takotsubo cardiomyopathy when they were evaluated for chest pain after admission to the hospital for treatment of COVID-19 infection; one on day 3 and the other on day 7 $[12,13]$.

\section{Electrocardiography (ECG)}

ST-elevation was seen in 11 patients $(42.3 \%)$. Other electrocardiography (ECG) patterns seen were sinus tachycardia, atrial flutter, atrial ectopics, atrial fibrillation, sinus tachycardia, QT- prolongation, T-wave inversion and non-specific ST-segment and T-wave changes. Cardiac troponins were elevated in 22 patients $(84.61 \%)$.

\section{Echocardiogram}

Echocardiography was a crucial diagnostic tool in all cases. Although most patients had typical takotsubo cardiomyopathy, four patients had inverted (reverse) takotsubo cardiomyopathy (15.38\%) [14-16]. Two others (7.69\%) had bi-ventricular involvement, one $(3.8 \%)$ had median takotsubo and another $(3.8 \%)$ had global takotsubo with apical sparing variant $[9,16,17]$. Ejection fraction was below $50 \%$ in all but one of the reported cases.

\section{Angiogram and ventriculography}

Conventional coronary angiography was done in nine patients $(34.61 \%)$. Eight of these patients did not have significant obstructive coronary lesions. One patient had significant lesions on the proximal left anterior descending (LAD) artery and first diagonal artery, and two drug-eluting stents were placed. However, since there were regional wall motion abnormalities in a different vascular territory than the ones supplied by obstructed arteries, the authors made a diagnosis of median takotsubo syndrome [9]. Another patient underwent Computed Tomography (CT) angiogram which showed absence of obstruction [18]. There was one patient who did not undergo coronary angiogram, but was found to have normal coronaries during autopsy [19]. The other patients did not undergo coronary angiography, either because they were too unstable to be shifted for the procedure, or because the treating team had already made a diagnosis and the angiogram would not have given any additional information. This would also avoid unnecessary viral exposure to the healthcare team and supporting staff. When ventriculography was done, it showed features similar to that seen on echocardiography.

\section{Other imaging modalities}

Cardiac magnetic resonance imaging (CMR) was done for one patient on day 7 of admission. It showed an improvement of systolic function from that at admission, with the persistence of mild hypokinesia at basal and mid-left ventricular segments [18]. 


\section{Management}

Management of most patients was done following local protocols for management of COVID-19 pneumonia and acute coronary syndrome or heart failure. Five patients received antiviral therapy consisting of a combination of lopinavir-ritonavir or darunavir-cobicistat $[15,18,19]$. One of them also received therapeutic plasma exchange [18]. Interleukin- 6 antagonists were received by two patients - one received tocilizumab while the other received sarilumab $[12,13]$. With such a small sample size, it is difficult to comment on the efficacy of any of these therapies in reversing takotsubo cardiomyopathy.

\section{Outcome}

Most patients had a positive outcome with complete or nearcomplete reversal of cardiac dysfunction at the time of discharge. Five deaths $(19.23 \%)$ were reported $[13,16,19]$. One of them had recovered cardiac function and died due to other factors. One patient was admitted at 38-weeks of gestation, and had an uneventful caesarean section delivery and was discharged on day 16 [10].

\section{Takotsubo cardiomyopathy in patients who tested COVID-19 negative}

There are two case reports of elderly women, who presented with chest pain, ST-elevation on ECG and elevated cardiac troponins, but were not found to have any obstructive coronary lesions on angiography [7,8]. Both ladies reported emotional stress related to the ongoing pandemic and social isolation, which was probably the trigger for takotsubo cardiomyopathy. A retrospective cohort study at cardiac catheterization laboratories in Ohio compared the incidence of Takotsubo cardiomyopathy in the pre-COVID and post-COVID era [11]. The incidence, which was $1.5-1.7 \%$ of all patients presenting with acute coronary syndrome in the pre-COVID era, had increased to $7.8 \%$ in the postCOVID era. All the patients in the study had a negative RT-PCR test for COVID-19, suggesting that the increase in incidence was

\section{Presentation}

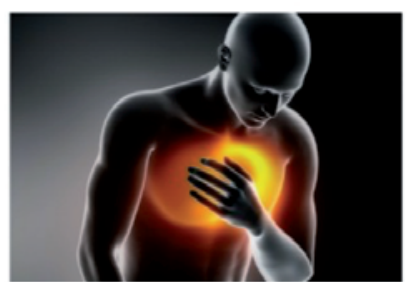

Chest pain(38.46\%)
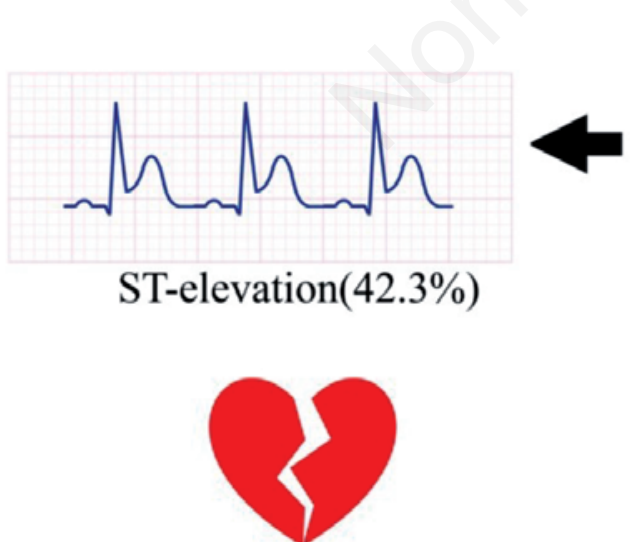

Mortality(19.23\%)
Diagnosis

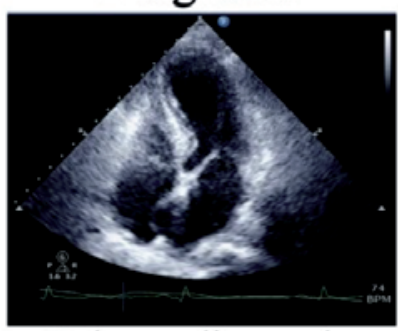

Echocardiography

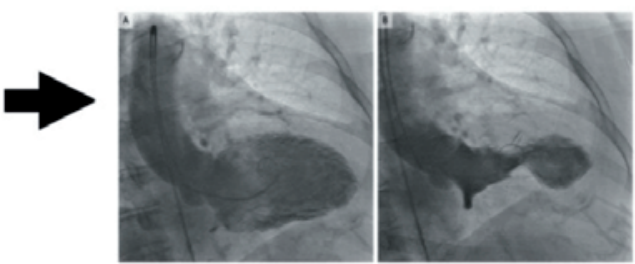

Angiography and ventriculography

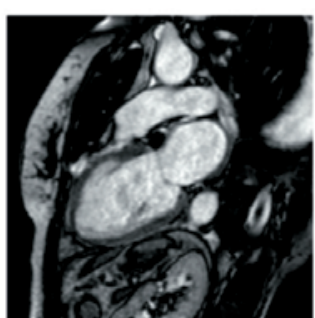

Cardiac MRI

Figure 1. Presentation and diagnosis of takotsubo cardiomyopathy in patients with COVID-19. 
due to factors other than infection itself. This study did not find a change in mortality rate or 30-day rehospitalization between the two time periods.

\section{Discussion}

At the end of 2019, the novel coronavirus was identified as the cause of a cluster of cases presenting with pneumonia in the city of Wuhan in the Hubei province of China. It rapidly spread throughout China and later, the world. On 30 $0^{\text {th }}$ January, 2020 the World Health Organization (WHO) declared this disease a Public Health Emergency of International Concern (PHEIC) and on Feb 2020, it officially named this coronavirus disease as COVID-19. COVID19 infection can be asymptomatic or symptomatic, and the severity of symptomatic infection can be mild to severe. Pneumonia is the most frequent serious manifestation of symptomatic infection and is characterized by fever, cough and dyspnea [20]. Several complications of COVID-19 have been described, such as, acute respiratory distress syndrome, cardiac arrhythmias, acute cardiac injury, pulmonary embolism, stroke, Guillain-Barre syndrome and secondary infections. Cardiovascular complications include thromboembolic events $(22 \%)$, heart failure $(12-23 \%)$, rhythm abnormalities (7-16\%), myocarditis $(8-12 \%)$, cardiogenic shock $(7 \%)$, pericarditis or effusions $(1-4 \%)$ and acute coronary syndromes $(<1 \%)$ [21]. A rare complication of COVID-19 is takotsubo cardiomyopathy, also known as stress cardiomyopathy, apical ballooning syndrome and 'broken heart syndrome'. The typical clinical presentation of takotsubo cardiomyopathy is indistinguishable from an acute coronary syndrome [5]. However, the disease is conspicuous for its absence of significant coronary lesions. Another remarkable feature of takotsubo cardiomyopathy is its association with emotional and physical stressful triggers. Although the exact mechanism of takotsubo cardiomyopathy is unclear, the catecholamine surge associated with these triggers almost certainly has a role in its pathogenesis.

Our review of the literature revealed two patterns of takotsubo cardiomyopathy associated with the COVID-19 pandemic. Some patients with COVID-19 infection had features of takotsubo cardiomyopathy. The stress response to the infection and hyper-adrenergic state may be responsible for this association, although viral myocarditis cannot be ruled out. The other group of patients presented with takotsubo cardiomyopathy as a result of the intense emotional stress caused by the global pandemic. Governments around the world have undertaken public health interventions to reduce the rate of transmission of COVID-19. One such measure is social distancing. Although effective in reducing the spread of infection and 'flattening the curve', it may be the cause of intense emotional distress, especially in the elderly who may find it difficult to use technology to stay in contact with their loved ones. This may act as a trigger for takotsubo cardiomyopathy in these individuals.

While the exact mechanism of takotsubo cardiomyopathy is unclear, there have been various postulates. Various theories such as multi-vessel coronary artery vasospasm, microvascular coronary impairments, catecholamine-induced myocyte injury and neurogenic myocardial stunning exist, but none have been proven conclusively [22]. Primary takotsubo cardiomyopathy is triggered by acute emotional and physical stress, while secondary takotsubo cardiomyopathy develops in patients hospitalized for other reasons (Figure 2). COVID-19 is a unique disease in that it can trigger takotsubo cardiomyopathy by both these mechanisms. The current pandemic, resultant social and economic instabilities, public health measures adopted by most governments including social distancing, inability to interact with loved ones and the anxiety of getting infected may all contribute to primary

\section{Primary Takotsubo Cardiomyopathy}

-Global pandemic

-Social and economic instability

-Unemployment

-Social distancing

-Disruption of usual routines

-Isolation from loved ones

-Anxiety of getting infected

-Thoughts of one's own mortality

\section{Secondary Takotsubo Cardiomyopathy}

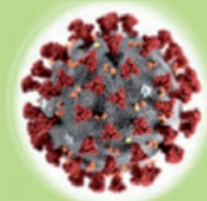

-Physiological response to infection

-Cytokine storm

-Catecholamine-inotrope use

-Coronary artery vasospasm

-Microvascular coronary impairments

-Neurogenic myocardial stunning

Figure 2. Association between takotsubo cardiomyopathy and COVID-19. 
takotsubo cardiomyopathy. On the other hand, patients admitted for treatment of COVID-19 infection, particularly those requiring intensive care, seem to be more prone to develop secondary takotsubo cardiomyopathy.

At the time of writing this review, there is one study that shows an increase in the incidence of takotsubo cardiomyopathy during the COVID-19 pandemic [11]. However, considering that in the past, the incidence of takotsubo cardiomyopathy has increased after natural calamities such as earthquakes, it is likely that the results of this study are valid and can be generalized [6]. As mentioned before, the exact mechanism of cardiac injury in takotsubo cardiomyopathy is unclear. SARS-CoV2 enters human cells by binding its spike protein to the membrane protein angiotensinconverting enzyme [23]. Viral RNA of Middle East respiratory syndrome coronavirus (MERS-CoV) and SARS-CoV, have been isolated from heart tissues of infected animals indicating cardiotropism [24]. However, the mechanism of takotsubo cardiomyopathy is most likely neurohormonal, rather than direct viral injury. Endomyocardial biopsy was performed in one patient with takotsubo cardiomyopathy and COVID-19 infection [18]. It showed diffuse T-lymphocytic inflammatory infiltrates with huge interstitial oedema and limited foci of necrosis, with no evidence of SARS$\mathrm{CoV}-2$ genome within the myocardium. Other mechanisms may also include procoagulant state, immune-mediated damage, endothelial injury and microvascular dysfunction. Viral RNA of Middle East respiratory syndrome coronavirus (MERS-CoV) and SARS-CoV, have been isolated from heart tissues of infected animals indicating cardio-tropism.

The evidence concerning optimal management of patients with takotsubo cardiomyopathy is unclear and most patients are managed with supportive care along the lines of management of heart failure. Also, it has to be noted that adrenergic stimulation is high in these patients, and therefore, exogenous catecholamine-inotropes such as dopamine, dobutamine, and norepinephrine can exacerbate neurocardiogenic injury and calcium overload in an already stressed myocardium. Therefore, the use of non-catecholamine inotropes such as milrinone and levosimendan may prove beneficial $[25,26]$. Patients with COVID-19 admitted in critical care may require hemodynamic support. Indeed, these catecholamine-inotropes may contribute to secondary takotsubo cardiomyopathy. Therefore, the treating team may consider levosimendan or milrinone as an alternative inotrope in situations where takotsubo cardiomyopathy is suspected. Betablockers may be beneficial in some cases of takotsubo cardiomyopathy, however, their use without invasive cardiac pressure gradient monitoring is controversial [27,28]. Therapeutic plasma exchange was employed for one patient as rescue therapy; however, it cannot be concluded whether it was that intervention which rescued the patient [18]. Plasma exchange is proposed to work by removing inflammatory cytokines such as interleukin-6 and may be especially beneficial at the onset of the cytokinerelease syndrome. Using IL-6 antagonists such as sarilumab and tocilizumab are also proposed to work by the same mechanism. However, there are several unknowns at this point - the optimal dose and duration of therapeutic plasma exchange, as well as IL6 antagonists, are yet to be determined. It is also unclear how reducing inflammatory mediators improves takotsubo cardiomyopathy- it may have a direct effect by reducing cardiac inflammation or may work indirectly by improving oxygenation and left ventricle filling pressures.

Takotsubo cardiomyopathy is considered to be a reversible form of cardiomyopathy with complete recovery of left ventricular function within weeks of presentation. This is reflected in the cases reviewed in this paper, with most patients having documented reversal of cardiac function at the time of discharge. Of the five patients who died, one had recovered cardiac function and succumbed due to other factors. It is difficult to say whether the other four patients died of cardiac or other causes.

Most likely, cases of takotsubo cardiomyopathy in COVID-19 are under-reported; either the diagnosis being missed completely, or being misdiagnosed as viral myocarditis. It is important to actively seek this diagnosis for two reasons. Firstly, once a diagnosis is made, invasive investigations such as angiography can be avoided, since they add minimal additional information and the management of takotsubo cardiomyopathy is largely supportive. This can also protect healthcare workers from unnecessary viral exposure during transportation and the procedure itself. Secondly, a diagnosis of takotsubo cardiomyopathy may urge the treating team to modify their management of critically-ill COVID-19 patients, especially with regards to limiting exposure to catecholamine-inotropes and considering the use of non-catecholamine inotropes. Because of these reasons, we recommend modification of protocols for evaluation of acute chest pain to include an echocardiography prior to coronary angiography in order diagnose takotsubo cardiomyopathy.

Reassuringly, takotsubo cardiomyopathy in itself seems to have a good prognosis in patients with COVID-19 infection. As more cases are reported, we may gain more insights into the optimal evaluation and management of this complication.

\section{Limitation}

There are several limitations to this review. The sample size is rather small and this affects the generalizability of these observations. It is possible that there are many unreported cases of takotsubo cardiomyopathy and if they were to be included, our observations may have been different. There is a chance for publication bias as more challenging cases and those with a positive outcome are likely to be reported. Also, we do not have complete details on inotrope requirement, ventilation, hospital stay and drug interactions-factors that may have affected the outcome $[29,30]$. Furthermore, there were no children included in the review. Lastly, the non-uniform drug therapies for COVID-19 due to changing recommendations make it difficult to comment on the efficacy of therapeutic interventions for takotsubo cardiomyopathy.

\section{Conclusions}

Takotsubo cardiomyopathy is a rare cardiovascular complication associated with the COVID-19 pandemic. With increasing cases of COVID-19, more cases of takotsubo cardiomyopathy are being reported. Takotsubo cardiomyopathy in COVID-19 can be primary takotsubo cardiomyopathy due to the emotional stress associated with the global pandemic, or secondary takotsubo cardiomyopathy due to COVID-19 infection. Less than half of the patients presented with chest pain. Although ECG abnormalities were commonly seen, ST-segment elevation was present in only $42.3 \%$ of the patients. Echocardiogram was the most important diagnostic tool and showed the classical findings of takotsubo cardiomyopathy or its variants. Management was mostly supportive and mortality was reported in $19.2 \%$ of patients. 


\section{References}

1. Li Q, Guan X, Wu P, et al. Early transmission dynamics in Wuhan, China, of Novel coronavirus-infected pneumonia. N Engl J Med 2020;382:1199-207.

2. Guan W, Ni Z, Hu Y, et al. Clinical characteristics of coronavirus disease 2019 in China. N Engl J Med 2020;382:1708-20.

3. Stokes EK, Zambrano LD, Anderson KN, et al. Coronavirus disease 2019 case surveillance — United States, January 22May 30, 2020. Morb Mortal Wkly Rep 2020; 69:759-65.

4. Akashi YJ, Goldstein DS, Barbaro G, Ueyama T. Takotsubo cardiomyopathy. Circulation. 2008;118:2754-62.

5. Prasad A, Lerman A, Rihal CS. Apical ballooning syndrome (Tako-Tsubo or stress cardiomyopathy): A mimic of acute myocardial infarction. Am Heart J 2008;155:408-17.

6. Sato M, Fujita S, Saito A, et al. Increased incidence of transient left ventricular apical ballooning (so-called 'Takotsubo' cardiomyopathy) after the mid-niigata prefecture earthquake. Circ J. 2006; 70:947-53.

7. Chadha S. "COVID-19 pandemic" anxiety-induced Takotsubo cardiomyopathy. QJM Mon J Assoc Physicians 2020;113:488-90.

8. Rivers J, Ihle JF. COVID-19 social isolation-induced Takotsubo cardiomyopathy. Med J Aust 2020;213:336-336.e1.

9. Nguyen D, Nguyen T, De Bels D, Castro Rodriguez J. A case of Takotsubo cardiomyopathy with COVID-19. Eur Heart J Cardiovasc Imaging 2020;21:1052.

10. Bhattacharyya PJ, Attri PK, Farooqui W. Takotsubo cardiomyopathy in early term pregnancy: A rare cardiac complication of SARS-CoV-2 infection. BMJ Case Rep 2020;13:e239104.

11. Jabri A, Kalra A, Kumar A, et al. Incidence of stress cardiomyopathy during the coronavirus disease 2019 pandemic. JAMA Netw Open 2020;3:e2014780.

12. Tsao CW, Strom JB, Chang JD, Manning WJ. COVID-19associated stress (Takotsubo) cardiomyopathy. Circ Cardiovasc Imaging 2020;13:e011222.

13. Dave S, Thibodeau JT, Styrvoky K, Bhatt SH. Takotsubo cardiomyopathy in a coronavirus disease-2019-positive patient: A case report. AA Pract 2020;14:e01304.

14. Solano-López J, Sánchez-Recalde A, Zamorano JL. SARS$\mathrm{CoV}-2$, a novel virus with an unusual cardiac feature: Inverted Takotsubo syndrome. Eur Heart J 2020;41:3106.

15. Faqihi F, Alharthy A, Alshaya R, et al. Reverse Takotsubo cardiomyopathy in fulminant COVID-19 associated with cytokine release syndrome and resolution following therapeutic plasma exchange: A case-report. BMC Cardiovasc Disord 2020;389:20.

16. Hegde S, Khan R, Zordok M, Maysky M. Characteristics and outcome of patients with COVID-19 complicated by Takotsubo cardiomyopathy: Case series with literature review. Open Heart 2020;7:e001360.

17. Minhas AS, Scheel P, Garibaldi B, et al. Takotsubo syndrome in the setting of COVID-19. JACC Case Rep 2020; 2:1321-5.

18. Sala S, Peretto G, Gramegna M, et al. Acute myocarditis presenting as a reverse Tako-Tsubo syndrome in a patient with SARS-CoV-2 respiratory infection. Eur Heart J 2020;41:1861-2.

19. Pasqualetto MC, Secco E, Nizzetto M, et al. Stress cardiomyopathy in COVID-19 disease. Eur J Case Rep Intern Med 2020;7:01718.

20. Wang D, Hu B, Hu C, et al. Clinical characteristics of 138 hos- pitalized patients with 2019 novel coronavirus-infected pneumonia in Wuhan, China. JAMA 2020;323:1061-9.

21. Mishra AK, Lal A, Sahu KK, et al. Quantifying and reporting cardiac findings in imaging of COVID-19 patients. Monaldi Arch Chest Dis 2020;90:1394.

22. Veillet-Chowdhury M, Hassan SF, Stergiopoulos K. Takotsubo cardiomyopathy: A review. Acute Card Care 2014;16:15-22.

23. Hoffmann M, Kleine-Weber H, Schroeder S, et al. SARS-CoV2 cell entry depends on ACE2 and TMPRSS2 and is blocked by a clinically proven protease inhibitor. Cell. 2020; 181:271280.e8.

24. Siripanthong B, Nazarian S, Muser D, et al. Recognizing COVID-19-related myocarditis: The possible pathophysiology and proposed guideline for diagnosis and management. Heart Rhythm 2020;17:1463-71.

25. Papanikolaou J, Tsolaki V, Makris D, Zakynthinos E. Early levosimendan administration may improve outcome in patients with subarachnoid hemorrhage complicated by acute heart failure. Int J Cardiol 2014;176:1435-7.

26. Mrozek S, Srairi M, Marhar F, et al. Successful treatment of inverted Takotsubo cardiomyopathy after severe traumatic brain injury with milrinone after dobutamine failure. Heart Lung J Crit Care 2016;45:406-8.

27. Santoro F, Ieva R, Ferraretti A, et al. Hemodynamic effects, safety, and feasibility of intravenous esmolol infusion during Takotsubo cardiomyopathy with left ventricular outflow tract obstruction: Results from a multicenter registry. Cardiovasc Ther 2016;34:161-6.

28. Isogai T, Matsui H, Tanaka $H$, et al. Early $\beta$-blocker use and inhospital mortality in patients with Takotsubo cardiomyopathy. Heart Br Card Soc 2016;102:1029-35.

29. Mishra AK, Lal A, Sahu KK, Sargent J. Cardiovascular factors predicting poor outcome in COVID-19 patients. Cardiovasc Pathol 2020; 49:107246.

30. Mishra AK, Sahu KK, George AA, Lal A. A review of cardiac manifestations and predictors of outcome in patients with COVID-19. Heart Lung 2020;49:848-52.

31. Roca E, Lombardi C, Campana M, et al. Takotsubo syndrome associated with COVID-19. Eur J Case Rep Intern Med 2020;7:001665.

32. Meyer P, Degrauwe S, Van Delden C, et al. Typical Takotsubo syndrome triggered by SARS-CoV-2 infection. Eur Heart J 2020;41:1860.

33. Taza F, Zulty M, Kanwal A, Grove D. Takotsubo cardiomyopathy triggered by SARS-CoV- 2 infection in a critically ill patient. BMJ Case Rep 2020;13:e236561.

34. Moderato L, Monello A, Lazzeroni D, et al. [Takotsubo syndrome during SARS-CoV-2 pneumonia: a possible cardiovascular complication].[Article in Italian]. G Ital Cardiol (Rome) 2020;21:417-20.

35. Dabbagh MF, Aurora L, D'Souza P et al. Cardiac tamponade secondary to COVID-19. JACC Case Rep 2020;21326-30.

36. Faqihi F, Alharthy A, Alshaya R, et al. Reverse takotsubo cardiomyopathy in fulminant COVID-19 associated with cytokine release syndrome and resolution following therapeutic plasma exchange: a case-report. BMC Cardiovasc Disord 2020;20:389.

37. Oyarzabal L, Gómez-Hospital JA, Comin-Colet J. Tako-tsubo syndrome associated with COVID-19. Rev Espanola Cardiol 2020;73:846. 